\title{
La importancia del primer strike para tener un buen rendimiento y una buena efectividad en los lanzadores abridores y relevos de la categoría juvenil
}

The importance of the first strike to have a good performance and a good effectiveness in the starting pitchers and relays of the youth category

Pedro Alexei Díaz Chapell. ${ }^{1}$, Dr.C Yasser Vázquez Alfonso. ${ }^{2}$, MsC. Carlos Batista Sotolongo. ${ }^{3} \&$ MsC. Velasteguí López Pablo ${ }^{4}$

Recibido: 17-07-2019 / Revisado: 19-08-2019 / Aceptado: 23-09-2019 / Publicado: 05-10-2019

Resumen. DOI https://doi.org/10.33262/concienciadigital.v2i4.1015

El béisbol está evolucionando en todos los órdenes, por lo que hay que despojarse de lo rutinario y pensar y obrar cada vez más alto a tono con los futuros compromisos que tiene este deporte y más aún con la entrada de los peloteros profesionales y el bate de madera, lo cual exige actualización. Se destaca como el objetivo de esta investigación la importancia de tirar el primer lanzamiento en la zona de strike, para tener una buena efectividad en los lanzamientos y un buen rendimiento. Este estudio me nos motivó a buscar y hacer una comparación de los lanzadores que lanzan el primer strike y de los que tienen problemas de concentración para hacerlo, además trataremos de hacer la comparación con el resto de los equipos del país para saber cómo está la situación actual de nuestra provincia. La población utilizada contó con la participación de 16 lanzadores del equipo de Béisbol categoría Juvenil de la EIDE de la provincia de Mayabeque, se emplearon los métodos científicos: la observación, la encuesta: tipo grupal y semi-estandarizada y el método sistémico estructural funcional.

Palabras claves: Strike., Efectividad., Lanzamiento., Béisbol., Lanzadores.

${ }^{1}$ EIDE “Antonio Jiménez Casadevalle” Cuba, padch@inder.cu

${ }^{2}$ Universidad de la Habana, Cuba, yalfos1@gmail.com

${ }^{3}$ Universidad de la Habana, Cuba, yalfos1@gmail.com

${ }^{4}$ Explorador Digital, Ambato, Ecuador, pablovelastegui@cienciadigital.org 


\section{Abstract.}

The baseball is evolving in all the orders, for what it is necessary to be robbed of the routine thing and to think and to work more and more high to tone with the future commitments that he/she has this sport and stiller with the entrance of the professional players and the wooden bat, that which demands bring up to date. He/she stands out as the objective of this investigation the importance of throwing the first launching in the strike area, to have a good effectiveness in the launchings and a good yield. This study motivated us to me to look for and to make a comparison of the pitchers that you/they throw the first strike and of those that have concentration problems to make it, we will also try to make the comparison with the rest of the teams of the country to know how the current situation of our county is. The used population had the participation of 16 pitchers of the team of Baseball Juvenile category of the EIDE of the county of Mayabeque, the scientific methods were used: the observation, the survey: type grupal and semi-standardized and the functional structural systemic method.

Key words: Strike., Effectiveness., Launching., Baseball., Pitchers.

\section{Introducción.}

El béisbol se ha ganado por derecho propio ser en nuestro país el deporte nacional por su arraigo y tradición. Se ha hecho acreedor de todos los títulos que confiere la AIBA en las diferentes categorías, menos el Clásico Mundial de Béisbol, no obstante, a estos triunfos no se debe subestimar a algunos países que se perfilan como poderosos contrarios, por ejemplo Japón y Corea del Sur.

Hoy en Cuba el béisbol es mucho más que el deporte nacional, forma parte de nuestra identidad, de nuestra idiosincrasia, totalmente en nuestra cultura deportiva inexplicable para alguien que no sea cubano, para aquellos que le hemos dedicado nuestros mejores años a cultivarlo y engrandecerlo sentimos con profunda satisfacción el desarrollo de nuestro béisbol revolucionario, pensando siempre que tenemos muchos errores que resolver y camino por andar si tenemos en cuenta sobre nuestros hombros pesa algo que nos ayuda cada día a ser mejores entrenadores de este gran deporte y es la crítica por parte de todo un pueblo que conoce, que disfruta, que critica, que apoya y que sigue tanto como a ningún otro deporte. Por todo esto estamos en la obligación de convertirnos en fieles estudiosos, científicos e incorporado cada vez más de la ciencia y la técnica en este mundo del béisbol debemos buscar nuevos caminos que nos permita encontrar resultados óptimos en el desempeño atlético. 
El desarrollo acelerado y vertiginoso del deporte actual, con tiempos y marcas cercanas al límite humanamente permisible, al accionar cada vez más veloz de los jugadores en los terrenos y canchas deportivas, hacen necesarios que los atletas tengan necesariamente que realizar una preparación multilateral para lograr altos resultados deportivos, esto solo es posible si desde bien tempranas edades se comienza a educar las diferentes capacidades con métodos y medios idóneos de acuerdo a la edad y su periodo de aprovechamiento en sus expresiones.

El estudio para iniciar esta investigación se realizó en el municipio de Güines en la provincia de Mayabeque, con una población de 16 lanzadores que constituye la muestra de lanzadores estudiados, de ellos 15 son derechos y 1 izquierdos, presentando una edad cronológica de 17 años y una edad deportiva de 8 años, 7 poseen el título de Técnico Medio en Cultura Física y cursan el 3er año de la licenciatura en Cultura física , 9 están en 3er año de técnico medio para profesores de Educación Física $(\mathrm{EPEF})$.

En una primera etapa no se tenía en cuenta si los lanzadores abrían los juegos en su primer inning dando strike o no lo importante era que el hombre caminara el juego y si tiraba duro mucho mejor así de esa forma podían obtener la victoria porque frenaba la ofensiva del equipo contrario. Ihosvany Gallego ex lanzador de los Industriales 1965-66.

Nosotros no mirábamos en aquel entonces la secuencia de los pitcheo como se hace ahora con ayuda de la tecnología pero si éramos un poco más inteligentes que los lanzadores de hoy en día, nosotros disfrutábamos el juego ya que cada bateador era un reto para los pitcher y eso ya te obligaba a pensar un poco más habían lanzadores que con su carácter ya resolvían el problema arriba del montículo mientras otro tenían que resolverlo con inteligencia y maldad porque no poseían una buena recta. Julio Romero ex lanzador de Pinar del Rio 198490.

Considero que nosotros no teníamos la tecnología que existe ahora y que hasta cierto punto se le hace el trabajo un poco más fácil el trabajo a los lanzadores pero siempre nos enseñaron que a los bateadores había que abrirle dando strike y tratar que ese primer bateador no llegara a la 1ra base y lo otro que nos enseñaron esos profesores que muchos eran analfabetos pero tenían vivencias del juego de beisbol era que teníamos que tener concentración para tener un buen resultado. Addiel Palma ex lanzador de Cienfuegos 1990- 2010. 
Considero que la tecnología ayuda al pitcher a resolver una situación que se presente durante el juego pero hay momentos que esa situación tenemos que resolverlo nosotros los lanzadores de conjunto con nuestros entrenadores y si considero que es de vital importancia el primer strike porque te ayuda a resolver todos los problemas durante el juego de béisbol. Yosvani Torres lanzador de Pinar del Rio.

En la actualidad los soportes tecnológicos y el estudio de la sabermetria nos ha dado información acerca del estado de los lanzadores en el caso de los juveniles y categorías escolares se hace a través de los campeonatos nacionales que los entrenadores van archivando, pero no es menos cierto que los lanzadores deben de abrir dando strike. Pedro Alexis Díaz entrenador de pitcheo de la EIDE.

\section{Análisis de los resultados.}

Tabla N 1: Resultados del promedio ganado y perdido según la salida que tuvieron los lanzadores.

\begin{tabular}{|c|c|c|c|c|c|c|c|c|}
\hline Nombre & J.L & J.I & J.C & J.R & J.G & J.P & PRO & J.S \\
\hline R. Bolaños & 4 & 0 & 0 & 4 & 0 & 1 & 0 & 0 \\
\hline M.Rodriguez & 3 & 1 & 0 & 2 & 1 & 1 & 500 & 0 \\
\hline K.Fundora & 10 & 4 & 1 & 6 & 2 & 1 & 667 & 2 \\
\hline O. GlL & 15 & 12 & 2 & 28 & 7 & 3 & 700 & 0 \\
\hline D.Ramirez & 11 & 0 & 0 & 11 & 4 & 0 & 1.000 & 6 \\
\hline A.Pereira & 18 & 0 & 0 & 18 & 1 & 1 & 500 & 3 \\
\hline Y.Gil & 12 & 12 & 3 & 0 & 7 & 2 & 800 & 0 \\
\hline M.Baez & 21 & 0 & 0 & 21 & 7 & 3 & 700 & 4 \\
\hline J.C.Hdez & 8 & 4 & 0 & 4 & 3 & 2 & 600 & 3 \\
\hline D.Napoles & 12 & 2 & 0 & 10 & 2 & 2 & 500 & 5 \\
\hline O.Soto & 6 & 1 & 0 & 5 & 3 & 1 & 0 & 2 \\
\hline A. Alvarez & 12 & 0 & 0 & 12 & 5 & 2 & 700 & 5 \\
\hline J.Bermudez & 14 & 3 & 1 & 11 & 3 & 3 & 500 & 4 \\
\hline R.Bolaños & 18 & 0 & 0 & 18 & 4 & 2 & 700 & 10 \\
\hline R.Cardosa & 15 & 0 & 0 & 15 & 4 & 2 & 600 & 6 \\
\hline Y.Meres & 12 & 12 & 8 & 3 & 6 & 3 & 800 & 1 \\
\hline
\end{tabular}

Leyenda: J.L- juegos lanzados, J.I- juegos iniciados, J.C- juegos completos, J.Rjuegos relevados, J.G- juegos ganados, J.P- juegos perdidos pro- promedio, J.Sjuegos salvados.

Como se podrá observar en la tabla 1 estas fueron las actuaciones que tuvieron los lanzadores en su campeonato nacional donde de manera individual se evaluaron las efectividades por salidas, así como el promedio de las actuaciones de calidad y las malas actuaciones, en el 
caso de los relevos al igual que los abridores también los contemplamos con los mismos parámetros de efectividad.

A continuación, les vamos a reflejar los resultados en números para evaluar la efectividad en entradas lanzadas frente a bateadores contrarios:

Tabla N 2. Resultados por entradas frente a bateadores durante el campeonato nacional de beisbol de la categoría juvenil.

\begin{tabular}{lcccccc}
\hline \multicolumn{1}{c}{ Nombre } & INN & V.B & B.E & AVE & PCL & WHIP \\
\hline R. Bolaños & 4 & 20 & 35 & 350 & 7.28 & 4.23 \\
M.Rodríguez & 15.1 & 59 & 69 & 254 & 3.52 & 3.52 \\
K.Fundora & 30.2 & 118 & 146 & 280 & 4.88 & 1.24 \\
O. GIL & 63.2 & 271 & 297 & 297 & 4.29 & 1.67 \\
D. Ramírez & 18 & 166 & 191 & 259 & 1.90 & 1.43 \\
A. Pereira & 34.2 & 145 & 168 & 269 & 3.12 & 1.64 \\
Y. Gil & 53.1 & 198 & 239 & 258 & 4.89 & 1.51 \\
M.Baez & 53.2 & 202 & 244 & 248 & 2.68 & 1.48 \\
J.C.Hdez & 34.1 & 133 & 154 & 286 & 2.45 & 1.47 \\
D. Nápoles & 35.2 & 100 & 126 & 229 & 3.56 & 1.71 \\
O. Soto & 23.2 & 87 & 92 & 345 & 5.03 & 2.63 \\
A. Álvarez & 22.2 & 89 & 98 & 222 & 3.36 & 1.34 \\
J. Bermúdez & 21.1 & 80 & 86 & 275 & 3.45 & 1.30 \\
R. Bolaños & 25.1 & 90 & 93 & 118 & 1.65 & 1.28 \\
R. Cardoza & 19.2 & 68 & 74 & 294 & 2.06 & 1.62 \\
Y. Meres & 23.1 & 79 & 88 & 277 & 2.00 & 1.97 \\
\hline
\end{tabular}

Leyenda: INNING- INNING donde tuvo participación, V.B- veces al bate, B.Ebateadores enfrentados, AVE- average ofensivo de bateadores contrarios. PCL- promedios de carreras limpias, WHIP-promedio de efectividad (Sumatoria de bases por bola más hit que permite el lanzador).

De esta forma se puede apreciar el rendimiento individual de cada lanzador durante el campeonato nacional de beisbol. Durante todo el torneo se fue haciendo énfasis en la 
importancia de tirar el primer lanzamiento en la zona de strike ya que de esta forma limitas las posibilidades del equipo contrario de que te hagan carreras. Además, en cuanto al desgaste físico te conservas un poco más ya que al realizar menos lanzamientos es menos gasto energético que debe realizar el lanzador.

Ejemplos de la sabermetria en cuanto a la información de los lanzadores.

Zona de strike. - reporte de los lanzadores

Lanzamientos por juegos de 9 entradas: 57,6 lanzamientos.

- Lanzamientos por comparecencia al bate: 3,5

VS DERECHOS (Conexiones fuertes: 13,8\%) (\%Strikes Calidad: 39,3\%) (\%Bolas: 40,2\%)

- $(55,4 \%)$ Recta (84-89 MPH) (Conexiones fuertes: 17,4\%) (\%Strikes Calidad: 37,4\%) (\%Bolas: 37\%): Utiliza mucho la zona afuera en strike.

- $\quad(25 \%)$ Curva (71-74 MPH) (Conexiones fuertes: 6,8\%) (\%Strikes Calidad: 54,5\%) (\%Bolas: 45,8\%): Utiliza mucho la zona baja adentro en strike. El 54\% de los lanzamientos los tira en zona mala. Los bateadores le hacen swing al $22,2 \%$ en zona mala. Utiliza mucho la zona baja adentro en bola.

Especialista en pitcheo. Capacidad para identificar las zonas y tipos de lanzamientos.

\section{BATEADOR \#56}

Bateador 56 (D): Conteo 2-2, recta (90 mph), zona alta en strike, R-2B/SS, $1 B$.

Bateador 56 (D): Conteo 0-1, slider (80 mph), zona afuera en strike, R-SS, $1 B$.

Bateador 56 (D): Conteo 0-1, curva (81 mph), zona baja en strike, R-3B, OUT.

Bateador 56 (D): Conteo 0-0, recta (93 mph), zona afuera en strike, RMF-1B/2B, $1 \mathrm{~B}$.

Bateador 56 (D): Conteo 3-2, recta (92 mph), zona centro, R-3B, OUT.

- Conocer las características del juego y las de su equipo de manera colectiva e individual.

Actualizarse sobre las nuevas tendencias del béisbol de alto nivel. Conocer sus debilidades, amenazas, fortalezas y oportunidades (y las del contrario). 


\section{Conclusiones}

- Los resultados obtenidos en el proceso investigativo desarrollado ponen de manifiesto que es de vital importancia la superación de los lanzadores juveniles para obtener un mejor rendimiento en el Béisbol.

- La concepción teórica que distingue la Teoría y Metodología del Entrenamiento Deportivo como su núcleo teórico y punto de partida para revelar aquellos aspectos trascendentes del proceso de preparación (en el ciclo anual del entrenamiento, del PDC y campeonato nacional de Béisbol); en ella se interpreta como se establecen las relaciones entre lo teórico y lo metodológico, al explicar, de manera ideal, el entrenamiento de los lanzadores. En esta concepción, se manifiesta lo sistémico como propiedad jerárquicamente superior.

- Manifiesta una contribución a la solución del problema científico develado y la comprobación parcial de la hipótesis de investigación declarada.

- Recomendaciones

- Valorar la posibilidad y pertinencia de extender este estudio a otros equipos participantes en los campeonatos nacionales de Béisbol.

- Proyectar un estudio similar con los lanzadores de Béisbol participantes en campeonatos provinciales y a nivel de base, fundamentalmente en relación con el mantenimiento de los niveles de rendimiento durante los campeonatos provinciales.

- Proyectar una línea investigativa relacionada con el control y la evaluación de la preparación de los jugadores de Béisbol de los campeonatos provinciales y particularmente, del desarrollo y mantenimiento durante la competencia.

\section{Referencias Bibliográficas.}

Álvarez, C. (2000). La investigación científica en la sociedad del conocimiento. Ciudad de La Habana: Editorial Academia.

Forteza, A. (1999). Direcciones del entrenamiento deportivo. Metodología de la preparación del deportista. Ciudad de La Habana: Editorial Científico-Técnica.

Ozolin, N. (1983). Sistema contemporáneo del entrenamiento deportivo. Ciudad de La Habana, Cuba: Editorial Científico-Técnica. 
Ortega, A. (2012). Metodología para la preparación de la fuerza rápida en atletas de Béisbol en la categoría Social. Efdeportes. Obtenido el 12 de enero de 2011, desde http://www.efdeportes.com

Pérez, I. (2008). Metodología para la evaluación del rendimiento competitivo en el Béisbol (ERC-Béisbol). Tesis de doctorado. Ciudad de La Habana: ISCF “Manuel Fajardo”. Estadísticas del Cinid de la Dirección Nacional De Béisbol y Comisión Nacional de Béisbol.

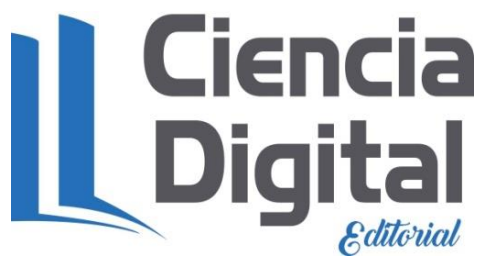


Para citar el artículo indexado.

Díaz Chapell, P., Vázquez Alfonso, Y., Batista Sotolongo, C., \& Velasteguí López, P. (2019). La importancia del primer strike para tener un buen rendimiento y una buena efectividad en los lanzadores abridores y relevos de la categoría juvenil. ConcienciaDigital, 2(4), 78-86. https://doi.org/10.33262/concienciadigital.v2i4.1015

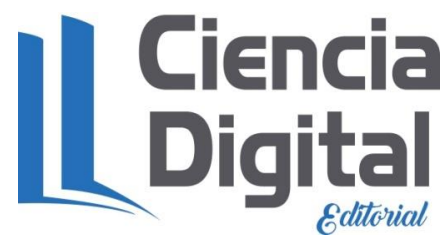

El artículo que se publica es de exclusiva responsabilidad de los autores y no necesariamente reflejan el pensamiento de la Revista Conciencia Digital.

El articulo queda en propiedad de la revista y, por tanto, su publicación parcial y/o total en otro medio tiene que ser autorizado por el director de la Revista Conciencia Digital.
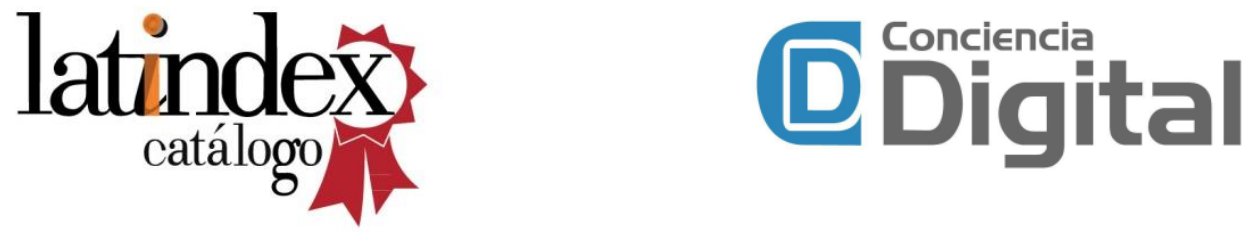